\title{
Novel sensing technology in fall risk assessment in older adults: a systematic review
}

\author{
Ruopeng Sun(i) and Jacob J. Sosnoff*
}

\begin{abstract}
Background: Falls are a major health problem for older adults with significant physical and psychological consequences. A first step of successful fall prevention is to identify those at risk of falling. Recent advancement in sensing technology offers the possibility of objective, low-cost and easy-to-implement fall risk assessment. The objective of this systematic review is to assess the current state of sensing technology on providing objective fall risk assessment in older adults.

Methods: A systematic review was conducted in accordance to the Preferred Reporting Items for Systematic Reviews and Meta-Analysis statement (PRISMA).

Results: Twenty-two studies out of 855 articles were systematically identified and included in this review. Pertinent methodological features (sensing technique, assessment activities, outcome variables, and fall discrimination/prediction models) were extracted from each article. Four major sensing technologies (inertial sensors, video/depth camera, pressure sensing platform and laser sensing) were reported to provide accurate fall risk diagnostic in older adults. Steady state walking, static/dynamic balance, and functional mobility were used as the assessment activity. A diverse range of diagnostic accuracy across studies (47.9\% - 100\%) were reported, due to variation in measured kinematic/ kinetic parameters and modelling techniques.

Conclusions: A wide range of sensor technologies have been utilized in fall risk assessment in older adults. Overall, these devices have the potential to provide an accurate, inexpensive, and easy-to-implement fall risk assessment. However, the variation in measured parameters, assessment tools, sensor sites, movement tasks, and modelling techniques, precludes a firm conclusion on their ability to predict future falls. Future work is needed to determine a clinical meaningful and easy to interpret fall risk diagnosis utilizing sensing technology. Additionally, the gap between functional evaluation and user experience to technology should be addressed.
\end{abstract}

Keywords: Geriatric, Older adults, Fall risk, Sensing technology

\section{Background}

Falls are the leading cause of accidental death and injury in older adults [1]. One in 3 older adults over the age of 65 and 1 in 2 over 85 years of age will experience a fall in the next year and a significant portion of those that fall will suffer an injury [2]. Given the adverse consequence of falls in older adults, considerable research has focused on identifying individual fall risk factors and targeted fall prevention [2-6]. This collective research has revealed that falls and fall-related injuries are

\footnotetext{
* Correspondence: jsosnoff@illinois.edu

Department of Kinesiology and Community Health, University of Illinois at

Urbana-Champaign, 301 Freer Hall, 906 S Goodwin Ave, Urbana 61801, USA
}

predictable and preventable with interventions targeting modifiable risk factors such as muscle strength, balance and mobility [7]. It is also maintained that effective fall prevention programs are cost effective and an appropriate method to maximize quality of life and maintain independence of older adults [7]. The first step to an effective fall prevention program is to identify those at risk of falling and then to determine the most appropriate interventions to reduce or eliminate falls [2].

While the American Geriatric society as well as the Centers for Disease Control and Prevention (CDC) recommends screening of fall risk for older adults at least annually by physicians [8], effective fall risk 
screening is still underutilized and not routinely integrated into clinical practice. There are several reasons for the lack of fall risk assessment in current practice ranging from overreliance on unreliable subjective measures, lack of cost-effective assessment technology and clinical time constraints.

Therefore, accurate, inexpensive, easy to administer fall risk assessments that can be undertaken regularly are warranted. Novel technology, such as inertial sensors, smartphone, low-cost video/depth camera, pressure sensors and motion ambient sensors, offer an alternative approach that can efficiently capture and analyze movement data and may provide an easy-to-implement objective fall risk assessment. Inertial sensor usually contains miniaturized accelerometers and/or gyroscopes that quantify movement pattern/abnormality by various time and frequency domain parameters. Low cost video/ depth sensing camera (i.e. Microsoft Kinect ${ }^{\mathrm{m}}$ ) provides marker-less 3D motion tracking of body joints by using its built-in and externally validated human skeleton modelling algorithms. It successfully eliminates the need for markers and calibration procedures characteristic of traditional motion capture, thereby enabling fast and patient-friendly 3D body motion analysis [9]. Low cost pressure sensing platform (Wii board, pressure sensitive insole / pad, etc.) provides critical information on postural stability as well as temporal pattern of stepping/gait $[10,11]$. Motion ambient sensing (Radar/Laser, etc.) technology can unobtrusively track movement of different body segments, and identify the movement abnormality in impaired individuals. Mobile phone has also been proposed as a potential instrument for balance/mobility tracking by using its built-in inertial sensor and/or camera [12-14].

To date, fall risk assessment technology has incorporated various screening tools, assessment activities, outcome variables, and fall discrimination/prediction models. There have been a few attempts to synthesize fall risk assessment using wearable sensing technology. A systematic review in 2013 by Howcroft and colleagues [15] focused entirely on inertial sensors use in fall risk assessment. It provided an overview of study methodologies, parameters derived and model effectiveness in faller prediction/discrimination, and concluded inertial sensors have the potential to provide objective fall risk assessment in older adults. Another review by Shany and colleagues in 2012 [16] provided a practical discussion on various issues concerning the use of using wearable sensors in a fall risk assessment context (i.e. whether it should or can be used in unsupervised environment). In a separated review, Shany et al. [17] raised concerns of over-inflated diagnostic accuracy in wearable sensorbased fall risk testing, primarily due to small sample size, questionable modelling decisions and inappropriate validation methodologies. However, there is a lack of knowledge about the use of other technologies (nonwearable) in fall risk assessment. Rapid technology advancements and additional research in recent years necessitates a timely systematic review of technology used in fall risk assessment. Consequently, the aim of this paper is to systematically evaluate the use of technology in performing fall risk assessments, and more specifically, to evaluate the test, sensor, algorithm effectiveness on predicting and/or discriminating older adult fallers from non-fallers.

\section{Methods}

\section{Search strategy and criteria}

This systematic review was conducted in accordance to the Preferred Reporting Items for Systematic Reviews and Meta-Analysis statement (PRISMA) [18]. Keyword search was performed in PubMed, Web of Science, Cochrane Library and CINAHL on May 2017. The search algorithm included all possible combinations of keywords (with wildcard characters and $\mathrm{MeSH}$ term) from the following 4 groups: (fall risk" OR fall predict" OR fall screen* OR fall assess*) and (walk OR locomot* OR ambulat* OR mobility OR gait OR balance OR postural OR posture OR reaction time OR strength) and (Aged OR geriatr* OR gerontol* OR senior OR elder* OR old*) and (acceler* OR inertia* OR gyro* OR wearable OR camera OR sens* OR phone* OR technolog* OR tool OR instrument*), Whereas articles with one or more of the following keywords were excluded: (exercise OR Intervention OR training OR free living OR daily OR activity). Reference lists from the identified publications were reviewed to identify additional research articles of interest.

Studies that met all of the following criteria were included in the review: 1) peer-reviewed publication in English language; 2) published since 2011 (to avoid redundant overlapping results with previous reviews conducted in 2012 [16] and 2013 [15]); 3) objective fall risk assessment conducted using commercially available technology (Inertial sensor, Video/depth Camera, Wii balance board, smartphone, pressure sensing insole, ambient sensing); 4) involvement of a geriatric population (based on a mean participant age greater than 60 years); 5) fall risk identified through accepted methods (physician screening, fall history, prospective fall incident tracking, or proven clinical fall risk assessment tool (Berg Balance Scale - BBS, Timed Up and Go - TUG, Short Physical Performance Battery - SPPB, Physiological Profile Assessment - PPA, Tinetti Performance Oriented Mobility Assessment - Tinetti, etc)); 6) the fall risk assessment involves a structured routine of movement sequence, and not free daily-living activity tracking; 7) evaluate the effectiveness (i.e. accuracy, specificity, 
sensitivity) [19] of the technology in identifying high risk faller. Studies were excluded from the review if they meet one or more of the following criteria: 1) Proof of concept on the technology use in movement tracking; 2) technologies that focused on fall detection; 3) studies that investigated fall risk solely in a neurological impaired population.

Titles and abstracts of the articles identified through keyword search were screened against the study selection criteria. Potentially relevant articles were retrieved for evaluation of the full text. The results of this search are shown in Fig. 1.

\section{Results}

\section{Study selection}

Figure 1 depicts the article identification and selection process. Eight hundred fifty-five unduplicated articles were identified through keyword and reference search. Eight hundred twenty-four articles were excluded after title and abstract screening. The remaining 31 articles were full text read. A total of 9 articles were excluded due to following reasons: 1) lack of diagnostic accuracy measures $(n=8), 2)$ duplicated cohort (i.e. studies based on identical samples) in assessment $(n=1)$. The remaining 22 articles were included in the review [10, 20-33].

\section{Study analysis}

Table 1 list the pertinent methodological features (faller identification method, participant demographics, sensor technology employed, sensor placement location if applicable, structured movement sequence used for fall risk assessment, outcome parameters used, modelling technique and performance for faller discrimination/prediction) from each included article.

\section{Faller identification}

Individuals at high risk of falls were identified with various techniques including retrospective fall history (11/ $22)$, prospective fall occurrence $(7 / 22)$, validated clinical assessment $(5 / 22)$, or physician exam $(1 / 22)$. Two studies combined the retrospective fall history and clinical assessments scores to identify high-risk fallers [34, 35]. The length of fall history recall (6 months to 1 year) and prospective fall occurrence follow up period (6 months to 2 years), as well as the number of falls chosen for faller classification (at least one fall, more than 2 falls, one fall requiring medical attention) also varied between studies.

\section{Sensor/activities used for fall risk assessment}

Inertial sensors were used in 17 investigations. Of those 17,11 utilized a sensor placed on the lower back area for quantifying center of mass movement $[10,22,24,26$,

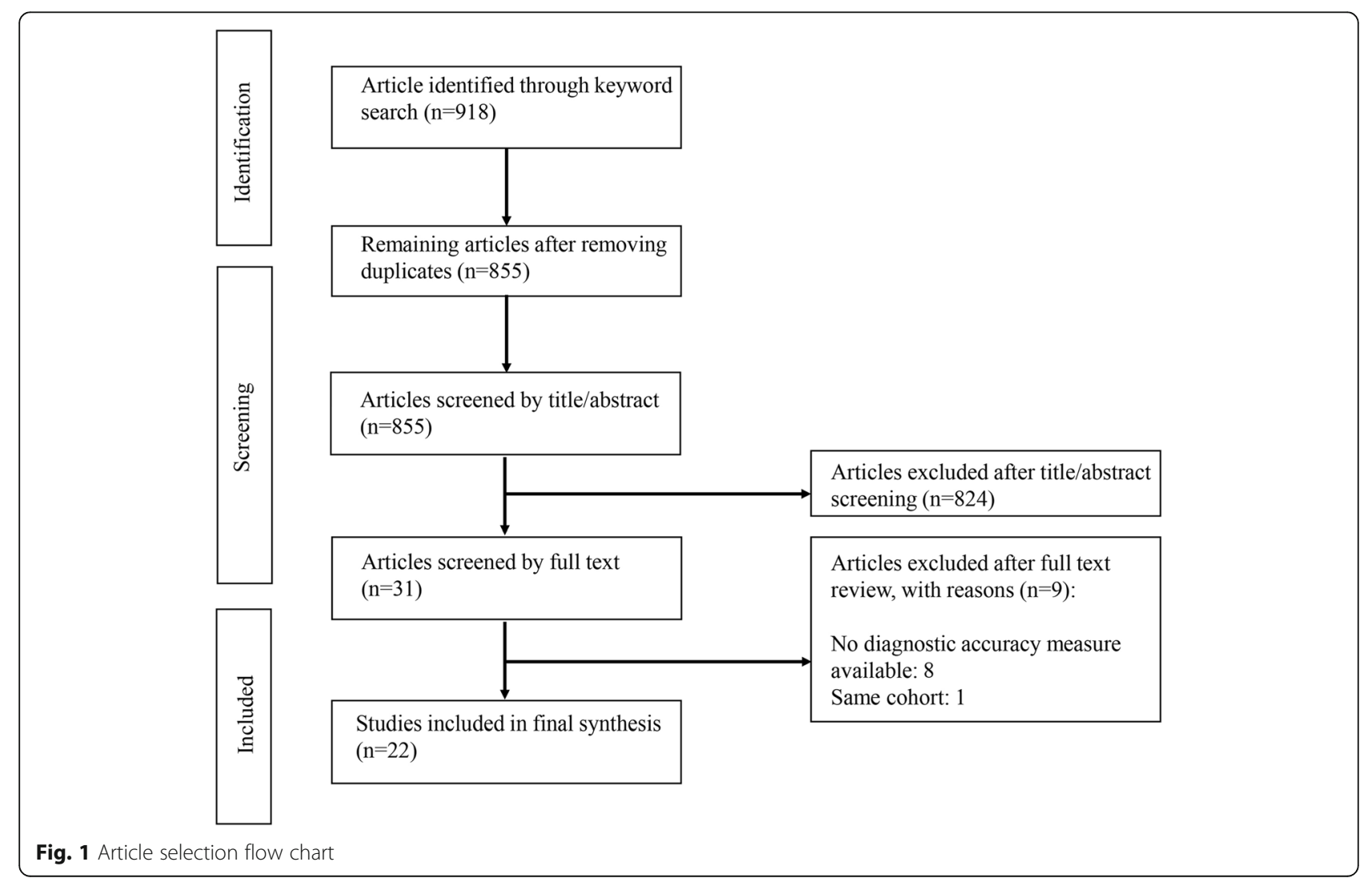




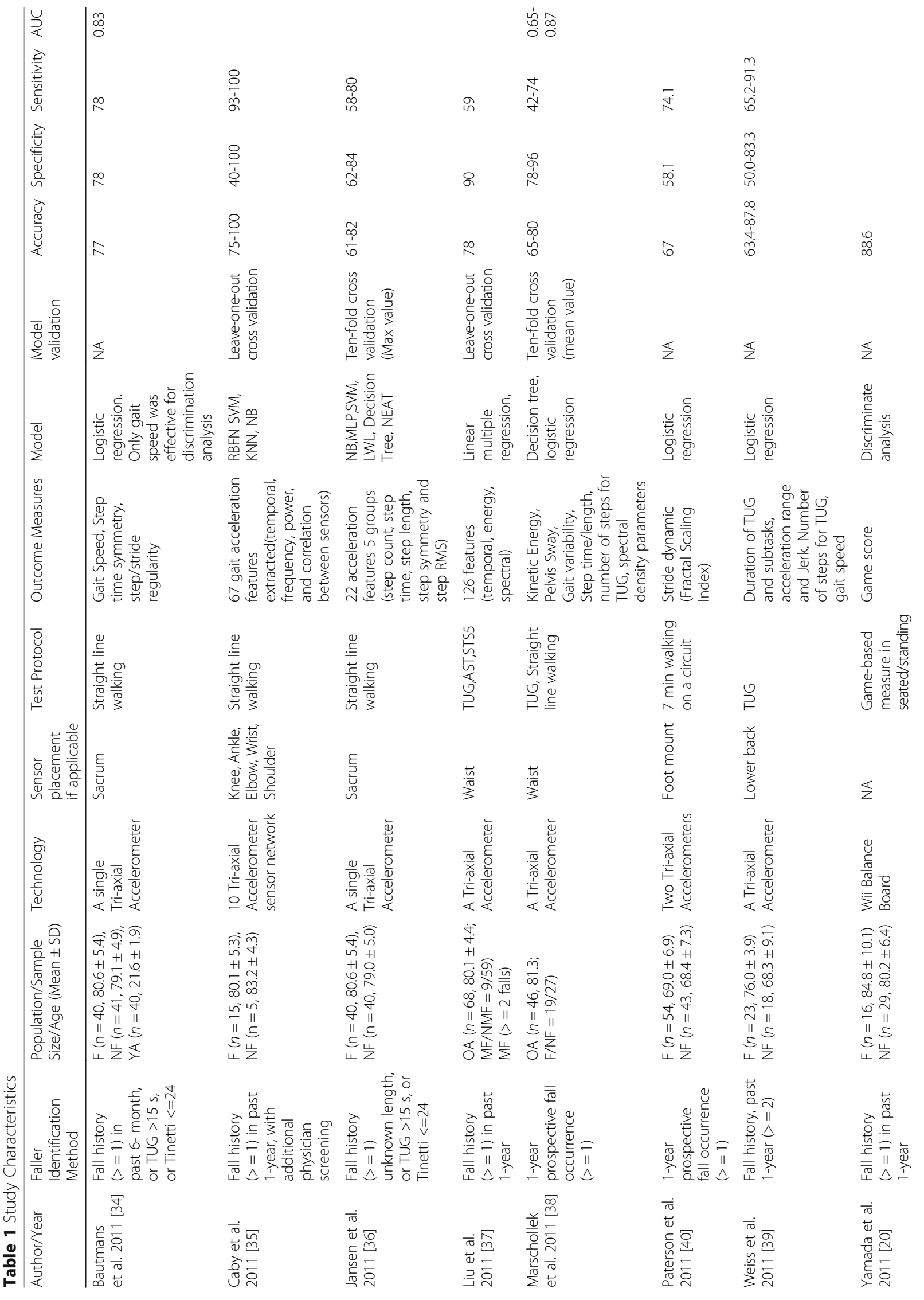




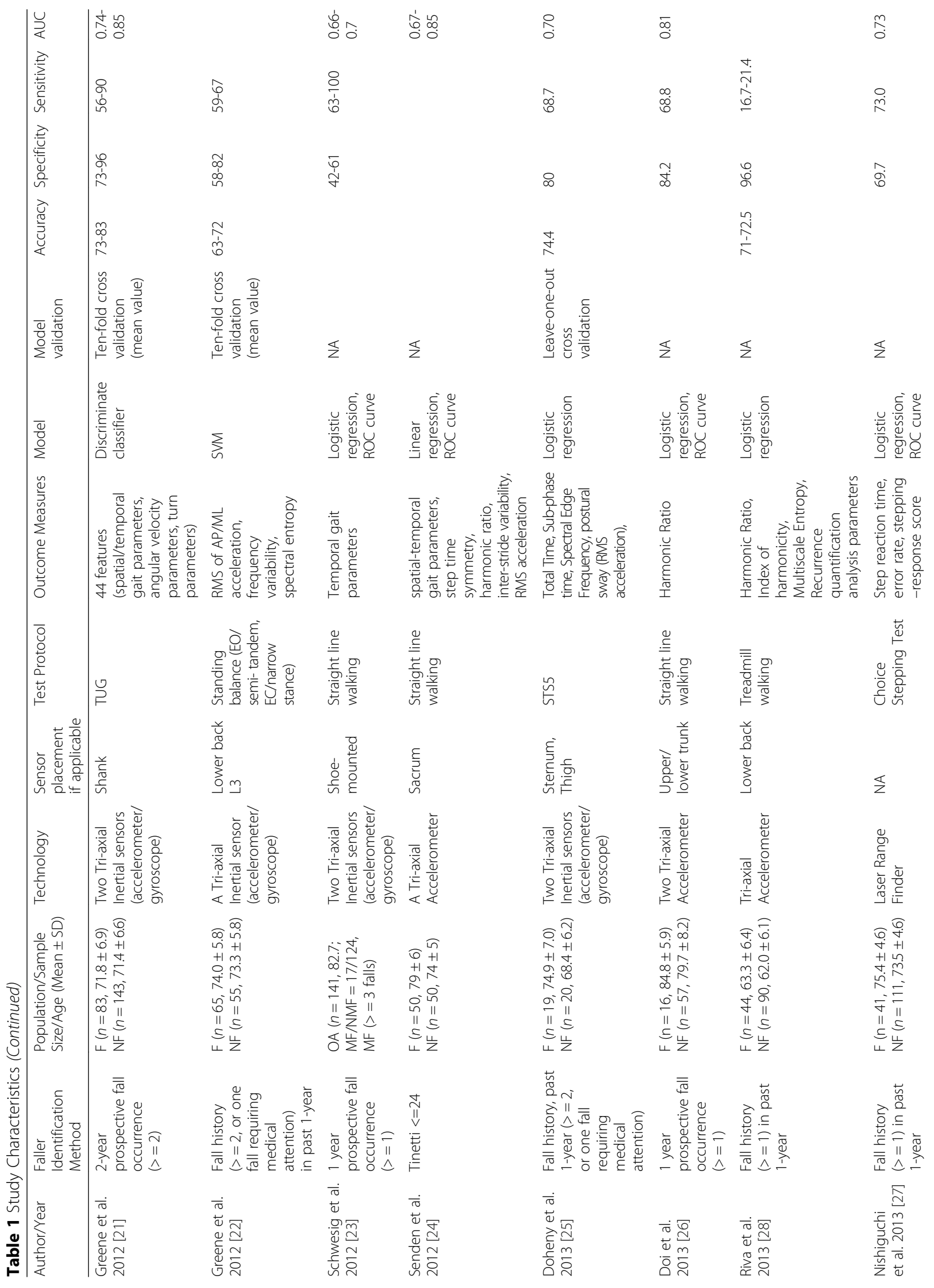




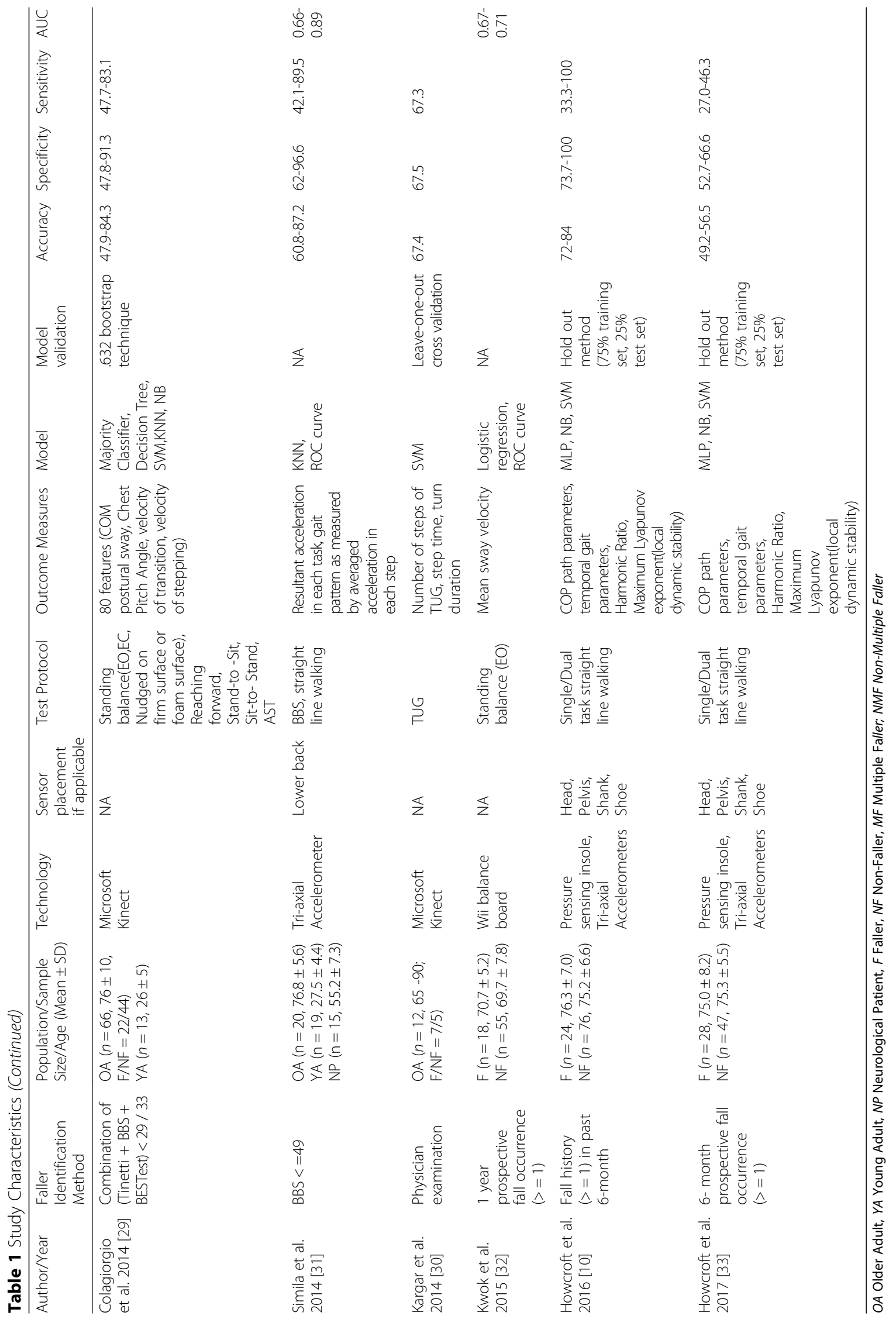


31, 33, 34, 36-39]. Six studies utilized sensors placed bilaterally on lower limb (foot/shoe/shank/ankle) for recording spatial/temporal gait parameters $[10,21,23$, $33,35,40]$, and 3 used a body sensor network ( $>3$ sensors on various anatomical landmarks: head, shoulder, wrist, elbow, knee, pelvis, ankle/shank) for quantifying limb and trunk kinematics [10, 33, 35]. Sternum sensor has also been used for quantifying trunk movement [25, 26], while thigh sensor was used in one study for quantifying the transition of sitting to standing [25]. In all studies, inertial sensors were secured on participant via belt, tape or band.

Given inertial sensor's unique benefit in unconstrained range of measurement, steady state walking (over ground/treadmill) was used as the primary assessment activity for 12 studies [10, 23, 24, 26, 28, 31, 33-36, 38, 40], while the Timed Up and Go (TUG) $(n=4)$ [21, 3739 ], five time sit to stand(5STS) $(n=2)[25,37]$, standing balance $(n=1)[22]$ and alternate step test (AST) $(n=1)$ [37] were also used in the assessment procedure.

Two depth camera (e.g. Kinect ${ }^{\mathrm{Tm}}$ ) based fall risk assessment studies were identified $[29,30]$. Due to its capture range $(0.5 \mathrm{~m}-4.5 \mathrm{~m})$, TUG [30], Standing balance, Sit-to-Stand transition and AST [29] were used as the fall risk assessment activities.

Two force-sensitive platform (Wii balance board) based fall risk assessment studies were identified [20, 32]. The tasks involved were standing balance [32] and seated/standing Wii balance gaming [20]. One laserbased fall risk assessment study used the choice stepping test as the fall risk screening activity [27].

Two studies done by the same research group utilized a pressure-sensing insole in conjunction with the inertial body-sensor network for steady-state walking assessment $[10,33]$.

\section{Outcome variables extracted}

For steady state walking assessment, 8 of 12 studies reported spatial-temporal gait parameters (step time, step length, etc) and/or its variability (SD, CV) [10, 23, 24, $33,35,36,38,40], 5$ of 11 studies reported harmonic ratio (an indicator for gait smoothness and overall stability) $[10,24,26,28,33]$. Other variables include gait speed [34], energy-related measures [38], frequencydomain measures $[28,38]$ local dynamic stability $[10$, 33], stride fractal scaling index [40] and gait symmetry $[10,24,33,34]$.

For test involving TUG assessment, extracted variables included time to complete the task and its subtasks (Sit-to-Stand, Stand-to-Sit, and turning) [37, 39], number of steps taken [30, 37, 39], cadence [37, 39],gait speed [37], temporal and spatial gait parameters and/or its variability $[21,30,37,39]$, acceleration/angular velocity/ jerk amplitude [21, 37, 39], energy-related measures [37], and frequency-domain measures [37].

For standing postural sway assessment, the inertial sensor based study reported RMS acceleration, frequency variability, and spectral entropy as the outcome variables [22], whereas the depth camera based study reported COM sway area, sway velocity, and the stochastic parameter of COM sway [29]. The Wii based study reported COP sway velocity as the outcome measures [32].

For fall risk assessment involving other dynamic tests (alternate step test, forward reaching, Stand-to-Sit, Sit-to-Stand transition, dynamic balance game, and choice reaction stepping), outcome measures included the duration of movement [25, 27, 37],velocity of movement [29], acceleration amplitude [25, 31], jerk amplitude [37], energy-related measures [37], and frequency-domain measures [25, 37], reaction time [27], error rate [27] and specific performance scores [20, 27].

\section{Modelling technique and effectiveness in faller discrimination}

A diverse collection of quantitative models/methods were employed to predict fall risk, which included: logistic regression, linear regression, Radial Basis Function Network classifier (RBFN), Support Vector Machine (SVM), Naïve Bayesian classifier, Multi-layer perceptron, Locally Weighted Learning, Decision Tree, Cluster analysis, k-Nearest Neighbor (kNN), neural networks, neuro evolution of augmenting topologies (NEAT), and discriminate analysis. Logistic regression was the most used modeling techniques $(N=10)$ for fall prediction modelling, although non-linear classification model was employed in recent publications.

Table 1 reports the effectiveness of diagnostic analysis (i.e. accuracy, sensitivity, specificity and area under curve for the ROC curve). Non-linear statistical classifier was primarily used in studies using inertial sensor and depth camera, due to the vast set of parameters extracted from the measured movement sequence. Whereas the Wii balance board and laser range finder measurement only provide a few features that require no advance modelling for fall risk identification. Due to the variation in assessment tools, movement routine, outcome variables extracted and modelling methods used, a diverse range of diagnostic performance was observed (Accuracy: 47.9-100\%, Sensitivity: 16.7-100\%, Specificity: 40-100\%, AUC 0.65-0.89). In term of classification technique, naive Bayesian classifier, SVM, discriminate classifier, $\mathrm{kNN}$, Decision tree, NEAT and logistic regression have been reported to achieve above $80 \%$ accuracy, although the direct comparison between classifiers cannot be achieved due to different tasks, parameters and populations. It is also worth noting that only $50 \%$ of studies were conducted with recommended model validation 
procedures such as Leave-one-out cross validation, Ten-fold cross validation, .632 bootstrap technique, and hold-out validation. Consequently, the diagnostic performance of studies without appropriate model validation could be over-inflated.

\section{Discussion}

This systematic review examined the existing evidence from 2011 to 2017 regarding sensor technology used in fall risk assessment in older adults. By measuring movement during selected structured movements (walking, stepping, sit-to-stand, stand-to-sit, TUG, BBS, etc.), the sensing technology has been shown as viable assessment tool for fall risk assessment. Overall, these devices provide an accurate, inexpensive, and easy-to-administer objective fall risk assessment.

Over half of the reviewed papers used the retrospective fall history and/or clinical assessment tools as the standard for identifying individuals at high risk for falls. However, even though widely used as standard methods, the clinical assessment tools (TUG, Tinetti, BBS, etc.) still do not achieve 100\% diagnostic accuracy. Furthermore, the retrospective fall recall may also suffer from the lack of reliability due to patient's poor recollection [41]. In addition, a history of falling may lead to gait pattern changes due to injury or fear of falling [17]. Thus, prospective fall occurrence tracking should be utilized in future fall risk assessment research with a follow-up period of at least 6 months after initial assessment.

A vast number of movement-derived variables were reported in the inertial sensor and depth camera based fall risk assessment investigations. They ranged from duration of movement to movement smoothness. Such diverse measurements resulted in a diverse range of prediction/discrimination accuracy across studies. A large pool of variables may not be clinical relevant, or confounding with other existing variables [17], thus a selection of proper variables based on research evidence is necessary. Additionally, lack of appropriate model validation procedures $(50 \%$ studies without cross-validation or holdout procedure) may yield over-inflated diagnostic accuracy, and are unlikely to maintain its reported performance during everyday use in relevant populations [17]. This observation highlights the need for proper guideline and standardized procedures of model construction/validation in future research [17].

It is worth noting that most if not all inertial sensor based fall risk assessment reviewed in this work used the sensor as a stand-alone recording device, thus require additional personnel to guide the user through the assessment routine, operate the system and interpret the data. Although with technological advances including increasing computing power, and integrated human interface devices (display, touch screen, voice command, etc.), this limitation may be minimized in the future. In contrast, the Kinect and Wii based systems, due to its compatible computer/gaming console, can provide automated interaction with the user, thus potentially allowing the user to complete the structured fall risk assessment without additional supervision. However, this unsupervised movement may raise other concerns such as safety and compliance.

Smartphone technology, although equipped with inertial sensors/camera, computing power and display to conduct an interactive assessment, has not been validated as a diagnostic tool for fall prediction [14]. However, numerous studies have validated its accuracy in balance and mobility tracking [12, 13], and several proof-of-concept have been proposed to investigate fall risk/ provide fall prevention intervention [42, 43].Other proof-of-concept studies that utilizing depth camera and radar sensing to provide in-home movement monitoring and fall risk assessment have also been reported in recent years $[6,44-46]$. In the long term, such ambient sensing technology may provide an unsupervised, automated fall risk screening tool in a community and/or assisted living settings. Several studies have also reported that IMU sensor and wireless pressure insole devices can continuously monitor daily-living activity, and derive gait parameters for diagnostic proposes [11, 47].

The primary goal of all existing technologies is to facilitate the identification of those at a risk of falling, and thus provide appropriate fall prevention intervention. Only limited investigation has been conducted to understand the seniors' acceptance in using the technology [43, 48, 49]. Overall, it has been suggested that older adults have a general interest in their health and fall risk [49], and selfcontrol, independence and perceived need for safety are important motivations to use the technologies [48]. Additionally, cost and privacy have also been reported as important elements that ensure continued use of technologies among older adults [48]. It is also important to consider that older adults do not want equipment to identify them as 'fallers' or in need of help [48]. Consequently, proper 'age-friendly' branding and user-centered design need to be considered in future research. Regarding the clinician acceptance of sensing technology use, minimal preparation time, simple interface and real time result display have been reported as key factors for continued use of technologies [50]. The disconnect between clinical functionality and user experience evaluation remains a significant gap and warrants attention.

\section{Conclusions}

To date, a wide range of sensor technologies have been utilized in fall risk assessment in older adults. Overall, 
these devices have the potential to provide an accurate, inexpensive, and easy-to-administer, objective fall risk assessment. However, the variation in measured parameters, assessment tools, sensor sites, movement tasks, and modelling techniques, precludes a firm conclusion on their ability to predict future falls. There is potential that these assessments can be undertaken regularly both in clinical and in non-clinical settings. To generate clinically meaningful and easy to interpret information, proper sensor-based predictors need to be identified. Additionally, a gap between clinical functionality and user experience remains.

\begin{abstract}
Abbreviations
5STS: Five Time Sit-to-Stand test; BBS: Berg Balance Scale; COM: Center of Mass; COP: Center of Pressure; KNN: k-Nearest Neighbor classifier; LWL: Locally Weighted Learning; MLP: Multi-layer perceptron classifier NB: Naiive Bayesian classifier; NEAT: Neuro Evolution of Augmenting Topologies; PPA: Physiological Profile Assessment; RBFN: Radial Basis Function Network classifier; ROC: Receiver Operating Characteristic; SPPB: Short Physical Performance Battery; SVM: Support Vector Machine; Tinetti: Tinetti Performance Oriented Mobility Assessment; TUG: Timed Up and Go
\end{abstract}

\section{Availability of data and materials}

All data generated or analyzed during this study are included in this published article and its supplementary information files.

\section{Authors' contributions}

Study concept, design: RS and JJS. Data acquisition: RS. Data analysis and interpretation: RS and JJS. Manuscript preparation: RS. Manuscript editing: RS and JJS. All authors have read and approved the final version of the manuscript.

\section{Ethics approval and consent to participate}

Not applicable

\section{Consent for publication}

Not applicable

\section{Competing interests}

The authors declare that they have no competing interests.

\section{Publisher's Note}

Springer Nature remains neutral with regard to jurisdictional claims in published maps and institutional affiliations.

Received: 16 October 2017 Accepted: 1 January 2018

Published online: 16 January 2018

\section{References}

1. Rubenstein LZ. Falls in older people: epidemiology, risk factors and strategies for prevention. Age Ageing. 2006;35(suppl 2):ii37-41.

2. Stevens JA. Falls among older adults —risk factors and prevention strategies. J Saf Res. 2005;36(4):409-11.

3. Podsiadlo D, Richardson S. The timed "up \& go": a test of basic functional mobility for frail elderly persons. J Am Geriatr Soc. 1991;39(2):142-8.

4. Mirelman A, Herman T, Brozgol M, Dorfman M, Sprecher E, Schweiger A, Giladi N, Hausdorff JM. Executive function and falls in older adults: new findings from a five-year prospective study link fall risk to cognition. PLoS One. 2012;7(6):e40297.

5. Buatois S, Miljkovic D, Manckoundia P, Gueguen R, Miget P, Vançon G, Perrin $P$, Benetos $A$. Five times sit to stand test is a predictor of recurrent falls in healthy community-living subjects aged 65 and older. J Am Geriatr Soc. 2008:56(8):1575-7.

6. Rantz M, Skubic M, Abbott C, Galambos C, Popescu M, Keller J, Stone E, Back J, Miller SJ, Petroski GF. Automated in-home fall risk assessment and detection sensor system for elders. The Gerontologist. 2015;55:S78-87.
7. Gillespie LD, Robertson MC, Gillespie WJ, Sherrington C, Gates S, Clemson LM, Lamb SE. Interventions for preventing falls in older people living in the community. Cochrane Database Syst Rev. 2012;9:11.

8. Stevens JA, Phelan EA. Development of STEADI: a fall prevention resource for health care providers. Health Promot Pract. 2013;14(5):706-14.

9. Geerse DJ, Coolen BH, Roerdink M. Kinematic validation of a multi-kinect v2 instrumented 10-meter walkway for quantitative gait assessments. PLoS One. 2015;10(10):e0139913.

10. Howcroft J, Lemaire ED, Kofman J. Wearable-sensor-based classification models of faller status in older adults. PLoS One. 2016;11(4):e0153240.

11. Di Rosa M, Hausdorff JM, Stara V, Rossi L, Glynn L, Casey M, Burkard S, Cherubini A. Concurrent validation of an index to estimate fall risk in community dwelling seniors through a wireless sensor insole system: a pilot study. Gait Posture. 2017;55:6-11.

12. Nishiguchi S, Yamada M, Nagai K, Mori S, Kajiwara Y, Sonoda T, Yoshimura K, Yoshitomi H, Ito H, Okamoto K. Reliability and validity of gait analysis by android-based smartphone. Telemedicine and e-Health. 2012:18(4):292-6.

13. Kim A, Kim J, Rietdyk S, Ziaie B. A wearable smartphone-enabled camerabased system for gait assessment. Gait Posture. 2015:42(2):138-44.

14. Roeing $\mathrm{KL}$, Hsieh $\mathrm{KL}$, Sosnoff JJ. A systematic review of balance and fall risk assessments with mobile phone technology. Arch Gerontol Geriatr. 2017;73:222-6.

15. Howcroft J, Kofman J, Lemaire ED. Review of fall risk assessment in geriatric populations using inertial sensors. Journal of neuroengineering and rehabilitation. 2013;10(1):91.

16. Shany T, Redmond S, Marschollek M, Lovell N. Assessing fall risk using wearable sensors: a practical discussion. Z Gerontol Geriatr. 2012:45(8):694-706.

17. Shany T, Wang K, Liu Y, Lovell NH, Redmond SJ. Are we stumbling in our quest to find the best predictor? Over-optimism in sensor-based models for predicting falls in older adults. Healthcare technology letters. 2015;2(4):79-88.

18. Moher D, Shamseer L, Clarke M, Ghersi D, Liberati A, Petticrew M, Shekelle P, Stewart LA. Preferred reporting items for systematic review and meta-analysis protocols (PRISMA-P) 2015 statement. Systematic reviews. 2015;4(1):1.

19. Lalkhen AG, McCluskey A. Clinical tests: sensitivity and specificity. Continuing Education in Anaesthesia Critical Care \& Pain. 2008;8(6):221-3.

20. Yamada M, Aoyama T, Nakamura M, Tanaka B, Nagai K, Tatematsu N, Uemura K, Nakamura T, Tsuboyama T, Ichihashi N. The reliability and preliminary validity of game-based fall risk assessment in communitydwelling older adults. Geriatr Nurs. 2011;32(3):188-94.

21. Greene BR, Doheny EP, Walsh C, Cunningham C, Crosby L, Kenny RA. Evaluation of falls risk in community-dwelling older adults using body-worn sensors. Gerontology. 2012;58(5):472-80.

22. Greene BR, McGrath D, Walsh L, Doheny EP, McKeown D, Garattini C, Cunningham C, Crosby L, Caulfield B, Kenny RA. Quantitative falls risk estimation through multi-sensor assessment of standing balance. Physiol Meas. 2012:33(12):2049-63.

23. Schwesig R, Fischer D, Lauenroth A, Becker S, Leuchte S. Can falls be predicted with gait analytical and posturographic measurement systems? A prospective follow-up study in a nursing home population. Clin Rehabil. 2012;27(2):183-90

24. Senden R, Savelberg H, Grimm B, Heyligers IC, Meijer K. Accelerometrybased gait analysis, an additional objective approach to screen subjects at risk for falling. Gait Posture. 2012;36(2):296-300.

25. Doheny EP, Walsh C, Foran T, Greene BR, Fan CW, Cunningham C, Kenny RA. Falls classification using tri-axial accelerometers during the five-times-sitto-stand test. Gait Posture. 2013;38(4):1021-5.

26. Doi T, Hirata $\mathrm{S}$, Ono R, Tsutsumimoto $\mathrm{K}$, Misu $\mathrm{S}$, Ando $\mathrm{H}$. The harmonic ratio of trunk acceleration predicts falling among older people: results of a 1-year prospective study. J Neuroeng Rehabil. 2013;10(1):7.

27. Nishiguchi S, Yamada M, Uemura K, Matsumura T, Takahashi M, Moriguchi T, Aoyama T. A novel infrared laser device that measures multilateral parameters of stepping performance for assessment of all risk in elderly individuals. Aging Clin Exp Res. 2013;25(3):311-6.

28. Riva F, Toebes MJ, Pijnappels M, Stagni R, van Dieen JH. Estimating fall risk with inertial sensors using gait stability measures that do not require step detection. Gait Posture. 2013;38(2):170-4.

29. Colagiorgio P, Romano F, Sardi F, Moraschini M, Sozzi A, Bejor M, Ricevuti G, Buizza A, Ramat S. Affordable, automatic quantitative fall risk assessment based on clinical balance scales and Kinect data. In: Engineering in medicine and biology society (EMBC), 2014 36th annual international conference of the IEEE. Piscataway: IEEE; 2014. p. 3500-3. 
30. Kargar BA, Mollahosseini A, Struemph T, Pace W, Nielsen RD, Mahoor MH. Automatic measurement of physical mobility in get-up-and-go test using Kinect sensor. Conference proceedings : Annual International Conference of the IEEE Engineering in Medicine and Biology Society IEEE Engineering in Medicine and Biology Society Annual Conference. 2014;2014:3492-5.

31. Simila H, Mantyjarvi J, Merilahti J, Lindholm M, Ermes M. Accelerometrybased berg balance scale score estimation. leee Journal of Biomedical and Health Informatics. 2014;18(4):1114-21.

32. Kwok BC, Clark RA, Pua YH. Novel use of the Wii balance board to prospectively predict falls in community-dwelling older adults. Clin Biomech. 2015;30(5):481-4.

33. Howcroft J, Kofman J, Lemaire E. Prospective fall-risk prediction models for older adults based on wearable sensors. IEEE Trans Neural Syst Rehabil Eng. 2017;25(10):1812-20

34. Bautmans I, Jansen B, Van Keymolen B, Mets T. Reliability and clinical correlates of 3D-accelerometry based gait analysis outcomes according to age and fall-risk. Gait Posture. 2011;33(3):366-72.

35. Caby B, Kieffer S, de Saint HM, Cremer G, Macq B. Feature extraction and selection for objective gait analysis and fall risk assessment by accelerometry. Biomed Eng Online. 2011;10(1):1.

36. Jansen B, Tan M, Bautmans I, Van Keymolen B, Mets T, Deklerck R: ACCELEROMETER BASED GAIT ANALYSIS multi Variate assessment of fall risk with FD-NEAT; 2011

37. Liu Y, Redmond SJ, Narayanan MR, Lovell NH. Classification between nonmultiple fallers and multiple fallers using a triaxial accelerometry-based system. In Engineering in Medicine and Biology Society, EMBC, 2011 Annual International Conference of the IEEE. IEEE; pp. 1499-1502.

38. Marschollek M, Rehwald A, Wolf K-H, Gietzelt M, Nemitz G, Meyer Zu Schwabedissen H, Haux R. Sensor-based fall risk assessment-an expert 'to go'. Methods Inf Med. 2011;50(5):420.

39. Weiss A, Herman T, Plotnik M, Brozgol M, Giladi N, Hausdorff JM. An instrumented timed up and go: the added value of an accelerometer for identifying fall risk in idiopathic fallers. Physiol Meas. 2011;32(12):2003-18.

40. Paterson K, Hill K, Lythgo N. Stride dynamics, gait variability and prospective falls risk in active community dwelling older women. Gait Posture. 2011;33(2):251-5.

41. Cummings SR, Nevitt MC, Kidd S. Forgetting falls. J Am Geriatr Soc. 1988;36(7):613-6.

42. Tacconi C, Mellone S, Chiari L. Smartphone-based applications for investigating falls and mobility. In: Pervasive computing Technologies for Healthcare (PervasiveHealth), 2011 5th international conference on. Piscataway: IEEE; 2011. p. 258-61.

43. Helbostad JL, Vereijken B, Becker C, Todd C, Taraldsen K, Pijnappels M, Aminian K, Mellone S. Mobile health applications to promote active and healthy ageing. Sensors. 2017;17(3):622.

44. Schoene D, Smith ST, Davies TA, Delbaere K, Lord SR. A Stroop stepping test (SST) using low-cost computer game technology discriminates between older fallers and non-fallers. Age Ageing. 2014;43(2):285-9.

45. Ejupi A, Brodie M, Gschwind YJ, Lord SR, Zagler WL, Delbaere K. Kinectbased five-times-sit-to-stand test for clinical and in-home assessment of fall risk in older people. Gerontology. 2016;62(1):118-24.

46. Ejupi A, Gschwind YJ, Brodie M, Zagler WL, Lord SR, Delbaere K. Kinectbased choice reaching and stepping reaction time tests for clinical and inhome assessment of fall risk in older people: a prospective study. Eur Rev Aging Phys Act. 2016;13(1):2.

47. van Schooten KS, Pijnappels M, Rispens SM, Elders PJ, Lips P, van Dieën JH. Ambulatory fall-risk assessment: amount and quality of daily-life gait predict falls in older adults. Journals of Gerontology Series A: Biomedical Sciences and Medical Sciences. 2015:70(5):608-15.

48. Hawley-Hague H, Boulton E, Hall A, Pfeiffer K, Todd C. Older adults' perceptions of technologies aimed at falls prevention, detection or monitoring: a systematic review. Int J Med Inform. 2014;83(6):416-26.

49. Ejupi A, Gschwind YJ, Valenzuela T, Lord SR, Delbaere K. A kinect and inertial sensor-based system for the self-assessment of fall risk: a home-based study in older people. Human-Computer Interaction. 2016;31(3-4):261-93.

50. Bergmann J, McGregor A. Body-worn sensor design: what do patients and clinicians want? Ann Biomed Eng. 2011;39(9):2299-312.

\section{Submit your next manuscript to BioMed Central and we will help you at every step:}

- We accept pre-submission inquiries

- Our selector tool helps you to find the most relevant journal

- We provide round the clock customer support

- Convenient online submission

- Thorough peer review

- Inclusion in PubMed and all major indexing services

- Maximum visibility for your research

Submit your manuscript at www.biomedcentral.com/submit 